absent. And, beside the phosphorus in the actual form of phosphates, milk contains further considerable quantity of phosphorus in the proteids and in nuclein." To the child who is not fed on milk, with so many factors necessary in nutrient-food, one may fail to be supplied by its artificial product. Hence, we may find that a diet prescribed with scientific skill, will fail sometimes, when success attends the other regulation of diet by an untutored mind. One mother says "my baby did well on Eagle Brand;" another, "that brand was no good for mine." One prefers 'Horlick's malted milk; another, Mellins'; some try them all without achieving any success. Why? Because the ingredient phosphorus is not given in sufficient quantity, while in the instances where prepared food succeeded, it may have been supplied from an independent source, as for instance, by the addition of good cow's milk. For M. Foster says, p. 630, "from a biological point of view, the phosphorus is no less important than carbon or nitrogen."

Manufacturers do not put up a food for the market devoid of carbon or nitrogen; these are blena'ed in exact proportions, but no account is taken of the salts. One says, when cow's milk is properly diluted the salts may be dismissed from separate consideraation; and yet the same will acknowledge that an infant may thrive on one woman's milk and suffer immediately from indigestion if put on another woman. "It has sometimes been necessary," he says, "to try a dozen." "It is possible," he says, " to vary the percentage of fat, sugar and proteids in milk to almost any degree desired, and to do this with very great accuracy." "At present," the same author says, "a separate modification of inorganic salts is not attempted." Though this style of preparing food may be of great value, when other important things are not overlooked, it is nothing to be relied on when the salts are regarded as being impossible to regulate. It only teaches the parents and physicians indolent reliance on manufactured articles in false security, instead of studying and practising the proper cooking of natural foods. And we need not believe that accuracy of proportion of carbon and nitrogen a positive necessity; for what was the anus given us if not for the elimination of unproportioned elements? Better leave it with a burden of work to do, rather than omit a small article of diet that is necessary for the proper performance of assimilation.

The numerous prepared foods for infants may all of them be useful for a change, though they may not contain the salts in the proper kind and quantities, for the carbon and nitrogen which they do contain. But life and health can not long be supported by them exclusively, and when the child is healthy we never think of using them.

In general, it is best in directing the diet of children to discard long courses of medicine, or drugs and prepared foods, and to try and select their diet from such foods and condiments as are generally found on the table, for we are then liable to get all those ele. ments of food that constitute the body. Then we shall stand a good chance of succeeding in improving malnutrition of any description, and of proving the benefit of our profession to a needing part of the community.

Physicians' Book=keeping. - The courte in Paris recently decided a case on the principle that a physician's books were sufficient evidence of his claim, unless there was manifest exaggeration.

\section{SOME THOUGH'TS ON THE CARE OF INFANTS AND CHILDREN.}

Presented to the Section on Diseases of Children, at the Forty-ninth Annual Meeting of the American Medical Association held at Denver, Colo, June 7-10, 1898.

BY J. A. WORK, M.D. ELKHART, IND.

Of all the young of God's vast creation of animated nature, none are so helpless as the newborn babe. The young of the lower order of animals can all early help themselves to their furnished food; not so with the human species; thus the demand for especial tender care; and how generously the mothers with natural affections bestow their aid and sympathy to the little weakling. Though perfect in its anatomic construction, yet as soon as breath enters the heretofore functionless lungs, the whole animal machinery of many members moves into new activity; and we can now declare, surely, that a new life exists. The first care that the infant's helpless nature demands is at the hands of the accoucheur and nurse, the responsibility largely resting on the former. The proper start in life, as in every other great undertaking, is an important factor to success.

It is fitly asserted, from a moral standpoint, that the beginning of the child's moral culture should precede its birth at least two generations; no less should be required of its physical training. Heredity should receive due consideration as to offspring. The very tender, pliable brain cells become arranged very early in life, in accord with its environments; habits of system, whether good or bad, become soon fixed and it becomes extremely difficult to change them. How deplorable to find a mother ignorant of and not com. petent to understand the first principles governing the physical interests of her offspring. This condition of mother, together with a careless or perchance impractical, inexperienced medical or physical adviser and nurse, presents anything but a flattering future for the helpless little creature of circumstances; however, we will take a more hopeful view and assume that all parties concerned in the well-being of the infant are adequate for the occasion.

With a mother ordinarily endowed, physically, morally and mentally, an accoucheur of good judgment, ever ready for emergencies, if any should arise, a nurse capable of obedience (at least) to all the details given her by her superior, the doctor; we are in a proper condition to safely assist the little personage to bis new and unexplored realm, suited to his wonderful possibilities. However, not a few infants are injured for life (and a miserable life, too) just at the last stage of the labor that gives them an existence. Sometimes this commiserable event is unavoidable; I refer first to the effect on the cranial viscera due to the employment of forceps, which injury of the brain the child scarcely, if ever, recovers from; second, the cervical portion of the spinal cord is often injured, or at least sometimes, by severe traction by the head and twisting of the neck, with or without instruments, when the shoulders are difficult of deliverance; this occurring usually after the head is delivered, and can be avoided by the accoucheur hooking his index finger into the axilla of the child, and thus employ traction which does not strain or injure the spinal cord

All things being equal, the first care of the infarit's body is to cleanse the surface of all extraneous or 
precipitate substances by the employment of some oleaginous agent, rich in glycerin. Fresh aseptic lard is the most efficient for this purpose of any substance I have yet employed. After the surface has been thoroughly anointed and the precipitate thus dissolved, then wipe with a soft cloth with as little friction as possible, and by not employing any kind of soap, the normal oil of the skin is preserved, and the above procedure adds to, rather than takes from, the normal condition of the surface, and renders it less liable to chafe.

The umbilical cord should be dressed in surgeon's aseptic cotton, and thus treated, as a rule it need not be disturbed, neither will it disturb, until it sloughs.

I do not recommend washing the infant's body oftener than once in two or three days, but during the interim of washings, gentle friction of surface of body with a soft cloth or hand, is good treatment. In washing, soft water should be employed.

Bands around the body should not be too tight, but should be snug enough to support the walls of the abdomen in case of distention. Clothing should be of the softest texture, and its conducting or non-conducting properties should be according to the conditions of the atmosphere. If mothers were taught and impressed with the importance of changing their children's clothing with the change of the atmosphere, I daresay many precious lives might be saved to the home and society, and much grief prevented. The child's clothing should be of equal warmth-that is, if possible, the surface temperature of all parts of the body should be the same and so maintained, and it is important to have clothing so constructed as to have equal pressure. This latter proposition should be also required of grown-up babies, and upon equal pressure, or rather no pressure, depends equal circulation and warmth, to a greater or less degree.

Face and hands should early be habituated to the atmosphere, not forgetting the self-evident fact that the infant is a new and separate being, and must do its own breathing, and should have a living chance to do it. The atmosphere should be of the purest quality, and not from under the bedcovering of the mother. Temperature of the nursery should be from $8 \tilde{j}$ to $9 \tilde{j}$ degrees at first, and gradually lowered to $7 \tilde{5}$ degrees; however, several days should elapse before reaching the lower temperature. Each individual case demands its own peculiar care, and the successful accoucheur must be an efficient nurse, or at least he must be competent to instruct his nurse efficiently, according to the needs of each particular case. The nurse is the physician's agent, or orderly, and should be so instructed as to readily know what constitutes a normal anatomic and physiologic babe, so that she may instantly discern any deviation from the standard; thus the need of trained and educated nurses in general obstetric and nursery practice.

The first few days of the infant's existence will reveal any anatomic or physiologic defect or weakness, and it is within the province of the nurse to notice any defect which has escaped the casual and hurried scanning of the busy attendant physician, and she should report at first opportunity. An effort should be made early in the child's life to correct defects, whether they be structural or functional, with a reasonable hope of success in a majority of cases.

One of the most irrational procedures in child culture is to neglect the physical while developing the mental; in fact, a proper development of being requires a strict observance of all the immutable laws govern. ing development or evolution. It is a sad mistake to withhold any of the vital stimuli from the feeble, whether it be of either body or mind. Pure outdoor air for the feeble infant or child, even at an expense of warmer clothing than is required for in-doors, is a wise investment, and will in time count on the credit side by bringing color to the cheeks and vigor to the priorly lax muscles, especially where the child is properly nourished. And I will note right here, that this department of the nursery is attended with more disaster than any other. There is a great diversity of toleration of food and powers of digestion and assimilation. This being true, it requires greater wisdom than is usually manifested to manage successfully the average child's larder.

If mothers can know the first symptoms of indiges. tion and take warning, know what to do and do it, if their sympathy did not overcome their judgment, much trouble could be averted. We must institute training schools for mothers, as we have for nurses, or else compel doctors to take time to instruct the young mothers, with authority to enforce obedience to the instructions. The deleterious results arising from the mistakes in the feeding of children are greater than in any other department pertaining to their physical culture. All things being equal, the mother's milk is the best suited to properly nourish the infant. Too often infants are weaned from their natural food too soon, without a reasonable cause, and substitute foods employed suddenly are dangerous. The proper and rational procedure in weaning a child, as a rule, is to wait until we notice an insufficiency of the natural food, and then gradually add the artificial, commencing with the smallest possible addition and increasing until we reach a sufficiency. The child will usually wean itself. As a rule, the Creator has wisely and abundantly provided nourishment for the infant; and substitution is unwarranted and is next to criminal. Next to the mother's milk, at our command, is fresh cow milk, if carefully and wisely administered.

Some contend that cow's milk should be sterilized. If it is not properly collected and contained, it is well to sterilize it, but if milked into sterilized containers and used soon after, there is no more necessity of going through the process of sterilization than there is to sterilize the mother's milk. In either case the fresh milk is sufficiently sterile, if the source is healthy.

\section{DISCUSSION.}

Dr. William C. Hollopeter, Philadelphia-First I would emphasize the fact that the first cleansing should be done with oil. An infant should not have water applied before several days after delivery. Several yearsago I had occasion to study this question particularly in reference to premature infants. I had two patients with deformed pelves, and I have delivered them each of three infants, one at six months and twenty-three days. The care of that infant consisted simply in keeping it in an equitable temperature with oil and not touching it with water, except about the nates with a cloth wrung out of hot water. Such an infant may have its death insured by improper treatment. Many infants die in the winter season by gradual cooling of the extremities, especially after being washed with water. I had a case two or three years ago in which I am sure the infant died from that sort of exposure, although it was in a well-to do family in the country. On the second or third day after its birth, at the beginning of the seventh month, it was being nursed by a trained nurse before a warm fire, wrapped up in a great wad of cotton on a cold bleak day. The cold draughts of air from the windows and doors going toward the 
fire, the side of the child from the fire was thoroughly chilled while the other side was warm. I was called out to bring my incubator on the third day. After the incubator was raised to a temperature of 85 degrees, on unwrapping the baby I found it cold and stiff. While the side toward the fire was red, the side away from the fire was cold and blue. Many infante born at full term after being washed with water are then put in a cradle, and especially in houses heated with a grate, we will get a chill of the side away from the fire, and bronchial trouble and other affections will arise which will cause its death. If the day is cold and chilly, $I$ intend to insist upon keeping the infants in a box, or any way so they are comfortable and will not chill.

Dr. Mary E, Green-Some one has said that two thirds of th the diseases of after life are due to the malnutrition of the first two weeks of infancy. I think that probably is largely true. One man has made the statement that nine-tenths of the children are fat starved. In view of all these statements that have come to us as practitioners, it behooves us to consider what the diet whould be. The Doctor very correctly said that the mothers should be educated along this line. I have so often noticed the patent foods recommended during the first two months of life. I believe that it is wrong to give the child any starchy food for the first six months of its life at least. I believe the child's food then should be cream, or the upper part of the milk, with water one-half. I like, what the Doctor said about the sterilization of milk. No child can remain healthy for a long time upon sterilized food. There is a lacking principle, so the child does not thrive on sterilized food. I think sterilization is unnecessary if perfect cleanliness is carried on in the preparation of the food. Some experiments have been carried on in giving children modified milk. But in all those laboratories absolute cleanliness is observed, and if as much cleanliness is practiced in preparing the usual food for infants, as good results will be obtained. All the attendants are obliged to wear duck suits, and perfect cleanliness is observed. The question of food is a very serious one up to the age of school life.

Dr. E. Stuver, Rawlins, Wyo.-I believe it was Holmes who once remarked that the education of a child ought to begin about two hundred years before it was born. The child ought to have a strong healthy mother, a mother who can provide an abundant supply of the nutriment that nature has intended the child should have during the first year of its life. I believe one of the most important points in securing good healthy children is to pay particular attention to the health of the child's mother; and after the child is born, if the mother has a disclination to nurse it, try to show her that is one of her duties as a mother to assume that responsibility. Further. more, I believe most of the patent foods do not supply what they should supply for the infant. Take for instance our condensed milks. A child may grow plump and apparently strong on condensed milk, but it is much in the same condition as the old beer drinker. Let him contract a serious disease and he has four or five times as many chances to die as a person in good health. The flabby, condensed-milk baby stands a good many more chances to die than the baby that has been fed on good natural mother's milk.

Dr. Brown of Illinois--The problem which I think especially interests practitioners in regard to feeding infants has occurred in the smaller towns where we are without milk laboratories and where it is absolutely impossible to have any form of modiffed milk. We are called into houses where children are dying of marasmus and malnutrition, where there is no ice in the house in the hottest summer months, where the milk is obtained from a milkman, carried several miles in hot cans on hot days before it reaches its destination. To make a modified milk out of that is entirely out of the question. I am in the habit of giving my better patients a formula for making a modified milk. But in the poor and ignorant classes it is impossible to give any instructions they can follow. We have to resort either to the patent foods or condensed milk. In choosing one of the two evils I choose condensed milk, because it is the only way to get a food in the smaller towns. I am in the habit of adding cream, because even if you get cream from milk in an unhealthy condition, carried around in tin cans three or four hours in the cities, the cream is less likely to be infected.

Dr, Mary E. GREen-I was going to make a remark about condensed milk. There are condensed milks and condensed milks. Some of those put up with sugar I think are objectionable, but there are condensed milks put up without sugar, and to those there is little objection. I have seen children brought up on such condensed milk and they have done well. Recently I attended the health exposition in New York. Three hundred cows were presented by Dr. Brush. It was an exhibition of the care of the cows and the milk. Dr. Brush furnishes the milk at 14 cents a quart. It is not sterilized or modifed in any way. The only thing about the dairy is absolute cleanliness. That is the one thing I think we should advocate more than anything else. Not long since I attended a dairy convention and $I$ heard a dairyman make the statement that he did not believe there were any dairymen furnishing milk to the cities in the summer who did not use some antiseptic, especially boracic acid or some form of borax. If that is so, $I$ think it is something physicians should know and we should fight against it. Whenever antiseptics are used they render the milk unfit for children. We want milk from a herd of cows rather than from one cow, but let it be clean.

Dr. R. B. GILBERT, Louisville, Ky. -The consideration of artificial food is secondary. If the child can not have its mother's milk, then we have to use something else. One thing is the condition of the mother furnishing the food for the infant. There are many times her milk is injurious. In a great many samples of milk this will be found to be true, if you examine it from day to day, and the doctor should do so continuously. The same thing may occur in the cow that furnishes the milk. I am glad to hear the statement that the milk should be taken from the whole herd rather than from any one cow. Not only that, but the food the mother eats has much to do. Food that contains pickles or anything of that kind, or food such as onions, will affect the milk. Mental anxiety will of ten modify the milk for several hours so the infant can not digest it. I do not know what the process is, but there is an excess of the casein and a diminishing of the peptones in a fit of anger or anxiety of any kind, or grief, as from a misfortune, for instance. It goes without saying that alcoholic stimulants all modify the milk. One case in particular comes to mind, of a child 6 months of age, that died while under my care. The mother went over in another part of the city to do a day's washing. On her return home she smoked an old strong pipe more than two-thirds of the distance home. Immediately after nursing the infant became limp, decidedly feeble, and I could hardly understand why it was so. It looked like it had been poisoned. On inquiry I found she had used two or three pipefuls of strong tobacco in smoking, and I attributed the death of the child to that. In reviewing the whole subject, you can not find any food, in my estimation, that is equal in any respect to the mother's milk.

\section{COLPOPERINEORRHAPHY AND THE STRUCTURES INVOLVED.}

DEDICATED TO DR. THOMAS ADDIS EMMET AND MR. LAWSON TAIT IN RECOGNITION OF THEIR LABORS IN THE SURGERY OF THE PERINEUM.

BY BYRON ROBINSON, B.S., M.D. CHICAGO, ILL.

Concluded from page 925.

Relaxed vaginal outlet, concealed lacerations or deep musculo-fascial tears of the pelvic floor, can not be toe forcibly brought to the notice of the physician as an important diagnostic indication for colpoperineorrhaphy. Kelly calls such, concealed relaxation. 